\title{
Variability of Solar Five-Minute Oscillations in the Corona as Observed by the Extreme Ultraviolet Spectrophotometer (ESP) on the Solar Dynamics Observatory/Extreme Ultraviolet Variability Experiment (SDO/EVE)
}

\author{
L. Didkovsky • A. Kosovichev • D. Judge · S. Wieman • \\ T. Woods
}

Received: 11 March 2012 / Accepted: 30 October 2012 / Published online: 23 November 2012

(C) The Author(s) 2012. This article is published with open access at Springerlink.com

\begin{abstract}
Solar five-minute oscillations have been detected in the power spectra of two six-day time intervals from soft X-ray measurements of the Sun observed as a star using the Extreme Ultraviolet Spectrophotometer (ESP) onboard the Solar Dynamics Observatory (SDO)/Extreme Ultraviolet Variability Experiment (EVE). The frequencies of the largest amplitude peaks were found to match the known low-degree $(\ell=0-3)$ modes of global acoustic oscillations within $3.7 \mu \mathrm{Hz}$ and can be explained by a leakage of the global modes into the corona. Due to the strong variability of the solar atmosphere between the photosphere and the corona, the frequencies and amplitudes of the coronal oscillations are likely to vary with time. We investigated the variations in the power spectra for individual days and their association with changes of solar activity, e.g. with the mean level of the EUV irradiance, and its short-term variations caused by evolving active regions. Our analysis of samples of one-day oscillation power spectra for a 49-day period of low and intermediate solar activity showed little correlation with the mean EUV irradiance and the short-term variability of the irradiance. We suggest that some other changes in the solar atmosphere,
\end{abstract}

Solar Dynamics and Magnetism from the Interior to the Atmosphere

Guest Editors: R. Komm, A. Kosovichev, D. Longcope, and N. Mansour

L. Didkovsky $(\bowtie) \cdot$ D. Judge $\cdot$ S. Wieman

Space Sciences Center, University of Southern California, Los Angeles, USA

e-mail: leonid@usc.edu

D. Judge

e-mail: judge@usc.edu

S. Wieman

e-mail: wieman@usc.edu

A. Kosovichev

Hansen Experimental Physics Laboratory, Stanford University, Stanford, USA

e-mail: akosovichev@solar.stanford.edu

T. Woods

Laboratory for Atmospheric and Space Physics, University of Colorado at Boulder, Boulder, USA

e-mail: tom.woods@lasp.colorado.edu 
e.g., magnetic fields and/or inter-network configuration may affect the mode leakage to the corona.

Keywords Solar p-modes · Helioseismology $\cdot$ Solar extreme ultraviolet irradiance . Spectrophotometer

\section{Introduction}

The frequencies of individual resonant acoustic modes (Claverie et al., 1979) that are excited by turbulent convection are stable within about $0.4 \mu \mathrm{Hz}$ on the time scale of the solar cycle because they correspond to intrinsic phase relations of resonant waves in the solar interior. Five-minute oscillations with frequencies centered at about $3.3 \mathrm{mHz}$ are trapped below the solar photosphere. However, several observations in photospheric and chromospheric lines, in the UV pass-bands, and in the coronal Fe XVI line $(33.5 \mathrm{~nm})$ demonstrate some leakage of these oscillations into the upper layers of the solar atmosphere (e.g. Judge, Tarbell, and Wilhelm, 2001; O'Shea, Muglach, and Fleck, 2002; McIntosh, Fleck, and Judge, 2003; Muglach, 2003, and De Pontieu, Erdelyi, and James, 2004). This leakage may be explained by the increased amplitude of the oscillations caused by the rapid density decrease (e.g. Gough, 1993), and/or by interaction with the network magnetic elements, which can channel the photospheric acoustic power to higher atmospheric layers at frequencies below the cutoff (Vecchio et al., 2007). For a discussion of these observations see Didkovsky et al. (2011).

Erdelyi et al. (2007) investigated the acoustic response to a single point-source driver. Malins and Erdelyi (2007) used a numerical simulation to show that widely horizontally coherent velocity signals from $p$-modes may cause cavity modes in the chromosphere and surface waves in the transition region, and that fine structures are generated extending from a dynamic transition region into the lower corona, even in the absence of a magnetic field.

A detection of the response of the corona to the observed photospheric low-degree $(\ell=0-3) p$-modes was reported by Didkovsky et al. (2011). The authors studied the oscillation power spectra of two six-day-long time series using the soft X-ray band-pass from the Extreme Ultraviolet Spectrophotometer (ESP) (Didkovsky et al., 2012) onboard the Solar Dynamics Observatory (SDO)/Extreme Ultraviolet Variability Experiment (EVE: Woods et al., 2012). The largest amplitude peaks in the five-minute spectral region were compared with the low-degree photospheric $p$-modes observed in Doppler velocity from the Birmingham Solar Oscillation Network (BiSON: Chaplin et al., 1998) and in visible-light intensity (red channel) from the SOHO/VIRGO instrument (Andersen, 1991; Fröhlich et al., 1997). This comparison showed that the frequencies of the coronal oscillations may deviate from the frequencies determined from the photospheric observations. This can be explained with the significant influence of the non-uniform distribution of the irradiance sources and variability of the upper atmosphere (Didkovsky et al., 2011). The mean standard deviation of the coronal frequencies from the photospheric $p$-mode frequencies was $\approx 3.7 \mu \mathrm{Hz}$ in the frequency range of $2.4 \mathrm{mHz}$ to $3.6 \mathrm{mHz}$, which is about two times larger than the uncertainty of the peaks in the power spectrum determined from the six-day time-series. This deviation was also confirmed by comparing the power spectra for two consecutive six-day time series with a spectrum for the combined 12-day period. The power spectrum for a single six-day time series showed more significant peaks with better correspondence to the photospheric $p$-mode spectrum than the combined 12-day spectrum. However, it was not clear whether the observed coronal oscillations were transmitted but distorted photospheric $p$-modes, or 
if these oscillations were excited in the corona by localized impulsive perturbation related to solar activity processes (e.g. modeling of Goode, Gough, and Kosovichev, 1992; Andreev and Kosovichev, 1995, 1998; Bryson, Kosovichev, and Levy, 2005). In this followup work we investigate whether the oscillations were excited in the corona by impulsive sources of solar activity (e.g. by flares) by studying the observational data during higher solar activity. If they are caused by solar activity, this study could reveal a correlation between the appearance of the coronal five-minute oscillations and this activity. In contrast to that, if the observed coronal oscillations (Didkovsky et al., 2011) were related to the transmission of photospheric $p$-modes through the upper atmosphere, the observations made during the higher solar activity may reveal that solar-irradiance variability and significant increases of the soft X-ray irradiance during solar flares add some solar "noise" to the data time series, and low-amplitude oscillation peaks in the power spectra may be masked by these noise peaks.

In this work we study the variability of coronal five-minute oscillations by analyzing 49 one-day power spectra for various solar activity observing conditions that range from the lowest solar activity observed during the SDO mission in the middle of May 2010 to intermediate solar-activity levels in 2011. As in Didkovsky et al. (2011), we use soft X-ray observations without spatial resolution in the zeroth-order channel of SDO/EVE/ESP.

\section{SDO/EVE/ESP Channels}

EVE (Woods et al., 2012) is one of three instrument suites on SDO. It provides solar EUVirradiance measurements that are unprecedented in terms of spectral resolution, temporal cadence, accuracy, and precision. Furthermore, the EVE program will incorporate physicsbased models of solar EUV irradiance to advance the understanding of solar dynamics based on short- and long-term activity of solar magnetic features. ESP (Didkovsky et al., 2012) is one of five channels in the EVE suite. It is an advanced version of the SOHO/CELIAS/SEM (Hovestadt et al., 1995; Judge et al., 1998) and is designed to measure solar EUV irradiance in four first-order bands of the diffraction grating centered around $19 \mathrm{~nm}, 25 \mathrm{~nm}, 30 \mathrm{~nm}$, and $36 \mathrm{~nm}$, as well as in a soft X-ray band from 0.1 to $7.0 \mathrm{~nm}$ (the energy range is 0.18 to $12.4 \mathrm{keV}$ ) in the zeroth-order of the grating. Each band's detector system converts the photo-current into a count rate (frequency). The count rates are integrated over 0.25 -second increments and transmitted to the EVE Science and Operations Center for data processing. An algorithm for converting the measured count rates into solar irradiance and the ESP calibration parameters is described by Didkovsky et al. $(2007,2012)$.

\section{Observations}

Our analysis of the ESP measurements was based on datasets for a long series of observations covering a much wider range of solar activity compared to the two six-day time intervals analyzed by Didkovsky et al. (2011). Due to the high sensitivity of the ESP zerothorder soft X-ray signal to solar activity and because of significant contamination of the power spectra in the five-minute band by the impulsive increases in irradiance during solar flares, we used data for the periods of low-to-intermediate solar activity, without strong solar flares. Based on these conditions, five data intervals were chosen (Table 1, Figure 1). Figure 1 shows these (thick horizontal bars) on the background of variations of soft Xray solar irradiance related to the increased phase of solar activity cycle. Intervals one to 


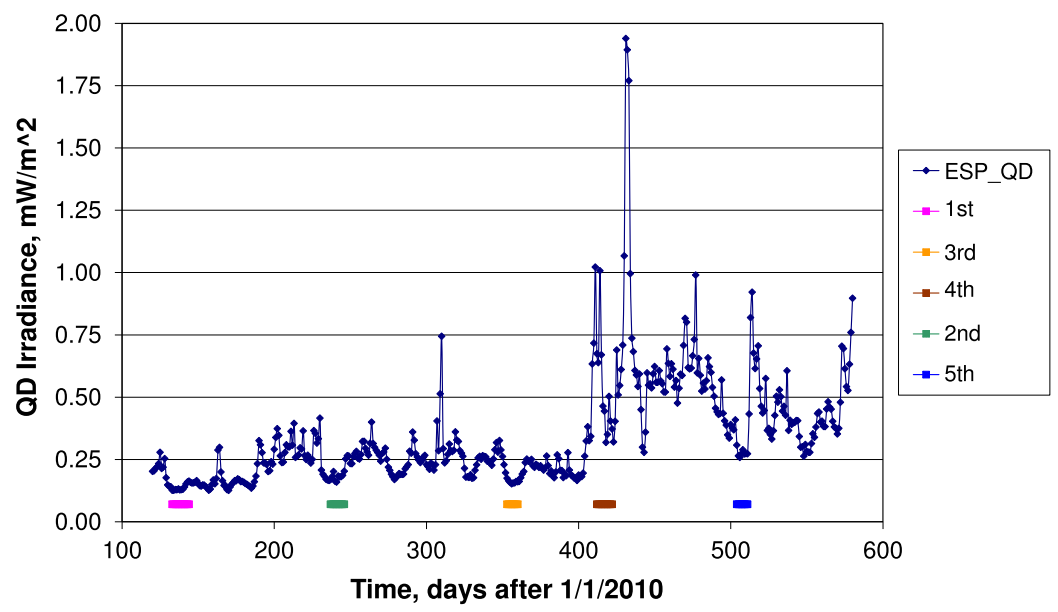

Figure 1 ESP zeroth-order $(0.1$ to $7.0 \mathrm{~nm})$ quad-diode (QD) soft X-ray irradiance measured for the first 461 days of the SDO mission, 245 days in 2010 and 216 days in 2011. The time intervals chosen are shown as wide horizontal bars below the irradiance curve. The mean number of analyzed days for each time interval was about ten, with the total number of days equal to 49 . The irradiance curve represents daily mean values while the actual cadence is 0.25 seconds.

three were chosen to represent the lowest periods of solar irradiance observed by SDO in 2010 , which are $0.137,0.181$, and $0.160 \mathrm{~mW} \mathrm{~m}^{-2}$. The fourth time interval was chosen between two periods of relatively high solar activity (Figure 1) with a mean solar irradiance of $0.531 \mathrm{~mW} \mathrm{~m}^{-2}$. The fifth time interval represents a return to relatively low solar activity with a mean irradiance of $0.276 \mathrm{~mW} \mathrm{~m}^{-2}$. Thus, these five time intervals cover a wide range of solar conditions for the periods of decreased solar activity. To establish a point of reference with our previous analysis, the first time interval matches the two six-day intervals analyzed previously (Didkovsky et al., 2011). Three of the time intervals (the second, third, and fifth) correspond to the lower-irradiance "spots" on the irradiance curve (Figure 1) with decreased solar activity, while the fourth time interval includes some C- and M-class solar flares to include some stronger disturbances of the solar atmosphere. Table 1 shows some details of the five-interval database. The lowest daily mean irradiance since the start of the SDO mission was $0.126 \mathrm{~mW} \mathrm{~m}^{-2}$ on 2010 DOY 133 , the lowest daily standard deviation (STD) was $3.31 \times 10^{-3} \mathrm{~mW} \mathrm{~m}^{-2}$ on 2010 DOY 135 , the highest flare-related mean irradiance value was $0.674 \mathrm{~mW} \mathrm{~m}^{-2}$ on 2011 DOY 047, and the highest flare-related STD of $0.505 \mathrm{~mW} \mathrm{~m}^{-2}$ was detected on 2011 DOY 055 (see Table 2).

\section{Data Reduction}

The data reduction was based on the ESP zeroth-order time series, whose original (level 0D) effective count rate (counts $\mathrm{s}^{-1}$ ) was corrected for energetic-particle events and temperature changes of dark counts (Didkovsky et al., 2012). The data were interpolated to eliminate short gaps (about two minutes total) that occur when the filters in the ESP filter wheel and observing modes change during routine daily calibration. The first step was to calculate a power spectrum for each of the 49 days. Then, a power-law curve for each spectrum was determined in a manner similar to that described by Didkovsky et al. (2011) for the best fit 
Table 1 Some details of the datasets. Columns (left to right) are time intervals, range of days within each time interval, days with GOES class B or larger solar flares, and solar flare class.

\begin{tabular}{|c|c|c|c|}
\hline $\begin{array}{l}\text { Time } \\
\text { interval }\end{array}$ & Range of days & $\begin{array}{l}\text { Days with solar } \\
\text { flares } \geq B 1\end{array}$ & GOES solar flare class \\
\hline \multirow[t]{4}{*}{1} & \multirow[t]{4}{*}{ 13-24 May 2010} & 13 May 2010 & B2.8 \\
\hline & & 22 May 2010 & B1.3 \\
\hline & & 23 May 2010 & B1.4, B1.3, B1.1 \\
\hline & & 24 May 2010 & B1.1 \\
\hline \multirow[t]{5}{*}{2} & \multirow[t]{5}{*}{25 Aug-3 Sep 2010} & 27 Aug 2010 & $\begin{array}{l}\text { B2.2, B1.6, B2.3, B4.7, B2.2, } \\
\text { B1.9, B1.3, B1.1, B2.2, B1.9, } \\
\text { B1.3, B1.1 }\end{array}$ \\
\hline & & 28 Aug 2010 & B1.0 \\
\hline & & 29 Aug 2010 & B1.7, B1.4 \\
\hline & & 30 Aug 2010 & $\mathrm{~B} 1.4, \mathrm{~B} 1.8$ \\
\hline & & 3 Sep 2010 & $\mathrm{~B} 2.8, \mathrm{~B} 1.2$ \\
\hline 3 & $19-26$ Dec 2010 & 19 Dec 2010 & $\mathrm{~B} 1.1$ \\
\hline \multirow[t]{2}{*}{4} & \multirow[t]{2}{*}{$16-26$ Feb 2011} & 16 Feb 2011 & $\begin{array}{l}\text { C2.0, M1.0, C2.2, C5.9, } \\
\text { C2.2, M1.1, C } 9.9, \text { C } 3.2, \\
\text { C1.0, M1.6, C } 7.7, \text { C1.3, } \\
\text { C1.1, C4.2, C } 2.8\end{array}$ \\
\hline & & 17 Feb 2011 & $\mathrm{C} 6.1, \mathrm{C} 2.3, \mathrm{C} 1.5, \mathrm{C} 1.0$ \\
\hline \multirow[t]{8}{*}{5} & \multirow[t]{8}{*}{ 19-26 May 2011} & 19 May 2011 & \\
\hline & & 20 May 2011 & $\mathrm{~B} 2.0, \mathrm{~B} 3.5, \mathrm{~B} 3.0, \mathrm{~B} 2.2$ \\
\hline & & 21 May 2011 & $\mathrm{~B} 1.6, \mathrm{~B} 1.1, \mathrm{~B} 2.7, \mathrm{~B} 1.5$ \\
\hline & & 22 May 2011 & $\mathrm{~B} 1.3, \mathrm{~B} 1.2$ \\
\hline & & 23 May 2011 & B1.9 \\
\hline & & 24 May 2011 & $\mathrm{~B} 2.0$ \\
\hline & & 25 May 2011 & $\mathrm{~B} 1.9, \mathrm{~B} 4.1, \mathrm{C} 1.1, \mathrm{C} 1.4, \mathrm{~B} 3.0$ \\
\hline & & 26 May 2011 & B $1.3, \mathrm{~B} 4.4, \mathrm{~B} 1.9$ \\
\hline
\end{tabular}

of the spectrum in the range of frequencies from $2.0 \mathrm{mHz}$ to $10.0 \mathrm{mHz}$, which includes our range of interest between $2.4 \mathrm{mHz}$ and $4.0 \mathrm{mHz}$,

$$
I 1_{i}(f)=A_{i} \times f^{-n_{i}},
$$

where $I 1$ is an array for the power-law spectral density, $i$ is the day number, $A_{i}$ is a constant, $f$ is the frequency, and $n_{i}$ is the power-law index. The third step was to calculate a running mean $[R M]$ curve that represents the local power change (local meaning in the five-minute band, for instance) in the power spectrum. Because we used one-day power spectra with a relatively low $(11.6 \mu \mathrm{Hz})$ frequency resolution and consequently a low confidence in the frequencies of individual peaks, the running-mean window of integration was chosen to be 13 seconds to reduce the influence of individual peaks in the power spectrum, but preserve the whole power change within the range of the power-law curve,

$$
I 2_{i}(f)=R M_{i}
$$

where $I 2$ is an array that represents the running-mean function. This is the standard IDL procedure, i.e. MEDIAN. The final step was to calculate the mean sum $\left[S_{i}\right]$ of the ratios 
Figure 2 ESP zeroth-order (0.1 to $7.0 \mathrm{~nm}$ ) power spectrum for 13 May 2010 (2010 DOY 133). The dashed (blue) line is a power-law curve $\left(I 1=0.18 f^{-0.88}\right)$, the red line $(I 2)$ is a running-mean curve. The two dotted vertical lines represent the edges of the power increase and the dashed vertical line shows the frequency of the increase maximum (see Table 4). The $S$-ratio for this example is 1.23 .

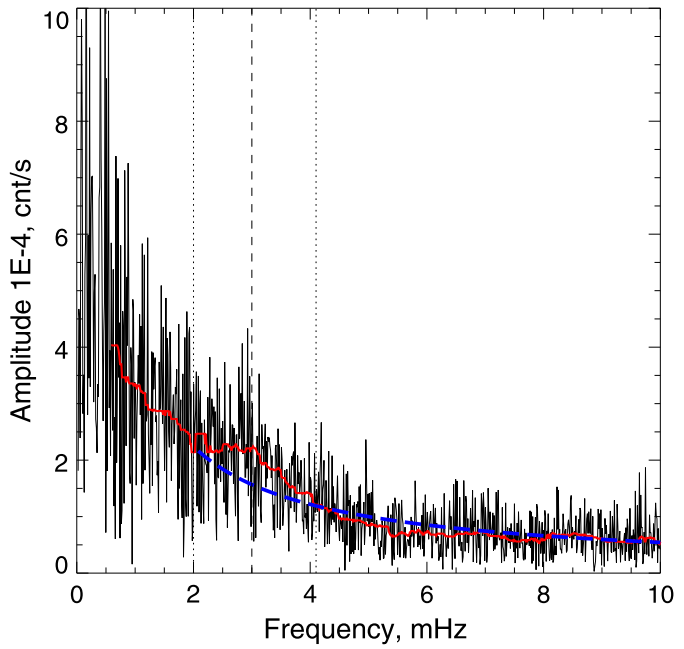

between the $I 2_{i}$ and $I 1_{i}$ over the frequency range of five-minute oscillations $\left(f_{1}=2.4 \mathrm{mHz}\right.$ to $f_{2}=4.0 \mathrm{mHz}$ ),

$$
S_{i}=\frac{\sum_{f_{1}}^{f_{2}} \frac{I 2_{f}}{I 1}}{m},
$$

where $m$ is the number of frequency bins within the frequency range. If the power spectrum showed an increase in the five-minute band, $S_{i}$ was greater than unity. As an example of this data-reduction algorithm, Figure 2 shows a power spectrum for 13 May 2010 with $I 1$ as a dashed (blue) line and $I 2$ as a red line. In addition to the power increase in the five-minute region, some peaks with frequencies above the cut-off frequency of $5.5 \mathrm{mHz}$ demonstrate increased amplitudes without the clear indication of increased power represented by the red line (Figure 2). For some other days the power increase in the frequency region from 6 to $10 \mathrm{mHz}$ is clearly observed. This increase may be similar to that found by Gurman et al. (1982) in the frequency range from 5.8 to $7.8 \mathrm{mHz}$ using SMM observations. An example of these increases in the five-minute region and above the cut-off frequency is shown in Figure 3.

\section{Results}

The results of the five-minute $S$-ratio calculations for the one-day power spectra are shown in Table 2 along with the changes of spectral irradiance, fluctuations of this irradiance (STD), and the maximum amplitude of the filter curve $I 2$ (Equation (2)) in the five-minute range. Columns (one to five) are days of observations in the (YYYY DOY) format, the daily mean irradiance $\left[10^{-1} \mathrm{~mW} \mathrm{~m}^{-2}\right]$, the daily standard deviation $\left[10^{-3} \mathrm{~mW} \mathrm{~m}^{-2}\right]$, the ratio $[S]$, and the filter $I 2$ maximum amplitude in the five-minute range $\left[10^{-4}\right.$ counts $\left.\mathrm{s}^{-1}\right]$. Each of the five time intervals is separated by an empty row. The $S$-ratio column in Table 2 is marked as N/A if the change of the spectral density in the power spectrum shows strong low-frequency variations that could be caused by strong changes of solar irradiance related to a solar flare, for instance. The gaps in the $I 2$ column are either for days in which the power spectra do not show any increase in the five-minute region (the $S$-ratio is $<1$ ) or for days where the 
Figure 3 ESP zeroth-order $(0.1$ to $7.0 \mathrm{~nm}$ ) power spectrum for the 23 May 2010 (DOY 142). The dashed line is a power-law curve $\left(I 1=7.010^{-4} f^{-1.1}\right)$, the red line $(I 2)$ is a running-mean curve. The two dotted vertical lines represent the edges of the power increase and the dashed vertical line shows the frequency corresponding to the increase maximum (see Table 4). The $S$-ratio for this example is 1.37 . Power increases above the cut-off frequency, at about $6-8 \mathrm{mHz}$, are more clearly visible than in Figure 2. To facilitate the comparison with Figure 2, the vertical scale is the same as in that figure.

Table 2 Comparison of the $S$-ratios (Equation (3)) in the five-minute band with the daily mean soft X-ray irradiance, the standard deviation of this irradiance (STD), and the filter I 2 maximum amplitude (Equation (2)).

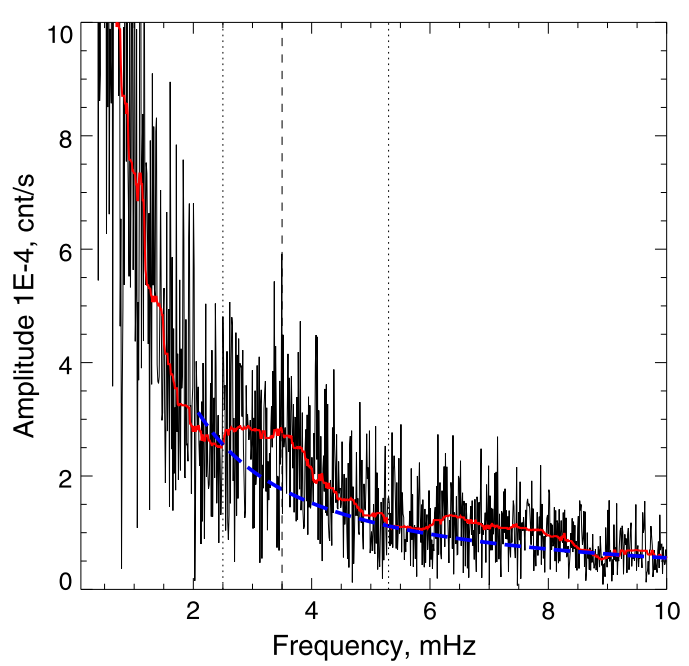

\begin{tabular}{|c|c|c|c|c|}
\hline $\begin{array}{l}\text { Day } \\
\text { [YYYY DOY] }\end{array}$ & $\begin{array}{l}\text { Daily mean, } \\
\text { irradiance }\end{array}$ & $\begin{array}{l}\text { Standard } \\
\text { deviation }\end{array}$ & $S$ & $I 2$ \\
\hline 2010133 & 1.26 & 7.54 & 1.23 & 2.3 \\
\hline 2010134 & 1.26 & 3.83 & 1.07 & 0.9 \\
\hline 2010135 & 1.29 & 3.31 & 1.14 & 1.4 \\
\hline 2010136 & 1.28 & 3.40 & 0.87 & \\
\hline 2010137 & 1.32 & 3.99 & 1.12 & 1.1 \\
\hline 2010138 & 1.28 & 3.47 & 1.06 & 1.1 \\
\hline 2010139 & 1.29 & 3.67 & 1.05 & 1.5 \\
\hline 2010140 & 1.30 & 3.90 & 1.07 & 0.9 \\
\hline 2010141 & 1.39 & 7.31 & 1.00 & 1.5 \\
\hline 2010142 & 1.52 & 9.58 & 1.37 & 2.9 \\
\hline 2010143 & 1.60 & 18.4 & 1.03 & 2.5 \\
\hline 2010144 & 1.63 & 11.7 & 1.01 & 2.5 \\
\hline 2010237 & 1.69 & 19.50 & 0.99 & \\
\hline 2010238 & 1.81 & 18.40 & 0.98 & \\
\hline 2010239 & 2.01 & 27.2 & N/A & \\
\hline 2010240 & 1.63 & 8.09 & 1.11 & 2.4 \\
\hline 2010241 & 1.59 & 6.68 & 1.05 & 2.4 \\
\hline 2010242 & 1.83 & 11.5 & 1.07 & 1.9 \\
\hline 2010243 & 1.80 & 6.96 & 0.98 & \\
\hline 2010244 & 1.81 & 5.43 & 1.03 & 1.7 \\
\hline 2010245 & 1.86 & 8.87 & 1.08 & 2.4 \\
\hline 2010246 & 2.03 & 13.6 & 1.02 & 3.1 \\
\hline 2010353 & 1.74 & 6.14 & 0.98 & \\
\hline 2010354 & 1.64 & 6.24 & 0.95 & \\
\hline 2010355 & 1.58 & 3.98 & 0.96 & \\
\hline
\end{tabular}


Table 2 (Continued.)

\begin{tabular}{|c|c|c|c|c|}
\hline $\begin{array}{l}\text { Day } \\
\text { [YYYY DOY] }\end{array}$ & $\begin{array}{l}\text { Daily mean, } \\
\text { irradiance }\end{array}$ & $\begin{array}{l}\text { Standard } \\
\text { deviation }\end{array}$ & $S$ & $I 2$ \\
\hline 2010356 & 1.52 & 3.41 & 0.78 & \\
\hline 2010357 & 1.55 & 4.47 & 0.95 & \\
\hline 2010358 & 1.56 & 4.91 & 0.88 & \\
\hline 2010359 & 1.62 & 5.09 & N/A & \\
\hline 2010360 & 1.61 & 4.68 & 0.90 & \\
\hline 2011047 & 6.74 & 288.0 & 1.24 & 225 \\
\hline 2011048 & 6.38 & 147.0 & 0.95 & \\
\hline 2011049 & 1.01 & 504.0 & N/A & \\
\hline 2011050 & 6.70 & 178.0 & 1.16 & 108 \\
\hline 2011051 & 4.64 & 75.9 & 0.97 & \\
\hline 2011052 & 4.44 & 112.0 & N/A & \\
\hline 2011053 & 3.18 & 25.1 & 0.96 & \\
\hline 2011054 & 3.50 & 42.2 & 0.93 & \\
\hline 2011055 & 5.03 & 505.0 & N/A & \\
\hline 2011056 & 4.05 & 36.9 & N/A & \\
\hline 2011057 & 3.72 & 60.6 & 1.08 & 28.4 \\
\hline 2011139 & 3.08 & 30.8 & N/A & \\
\hline 2011140 & 2.65 & 9.3 & 0.95 & \\
\hline 2011141 & 2.58 & 6.37 & N/A & \\
\hline 2011142 & 2.72 & 9.66 & N/A & \\
\hline 2011143 & 2.89 & 10.6 & N/A & \\
\hline 2011144 & 2.72 & 12.8 & 1.02 & 3.3 \\
\hline 2011145 & 2.73 & 32.1 & N/A & \\
\hline 2011146 & 2.73 & 20.1 & N/A & \\
\hline
\end{tabular}

$S$-ratio in the $S$-ratio column is marked as N/A because of strong contamination from solar flares. As Table 2 shows, the largest number of these contaminated spectra corresponds to the fourth and fifth time intervals with significantly higher solar activity (see the STD in Table 2) than during the first three time intervals.

\subsection{How Solar Activity Affects the Oscillations in the Corona}

To analyze how the oscillations in the five-minute band represented by the $S$-ratios (Table 2) and by the amplitude of the filter curve $I 2$ (Equation (2)) are related to the changes of the observing conditions, two parameters of these conditions, the daily mean irradiance and standard deviation of the irradiance, were compared with the changes of the $S$-ratios and the maximum amplitude of filter $I 2$. Table 2 shows that $S$-ratios higher than unity are detected for the first, second, and fourth time series. Figures 4-6 show these time series. Column I 2 in Table 2 for the amplitude of the filtered curve and the open circles in Figures 4, 5, 6 do not show any negative amplitude compared to the power-law curve (Equation (1)) for the days for which the $S$-ratio is lower than unity. The filtered curve $I 2$ (Equation (2)) for these days is below the spectral density $I 1$ (Equation (1)) and represents only noise. The $S$-ratios (Table 2) are plotted according to the linear scale shown at the right-hand side of the plots. 


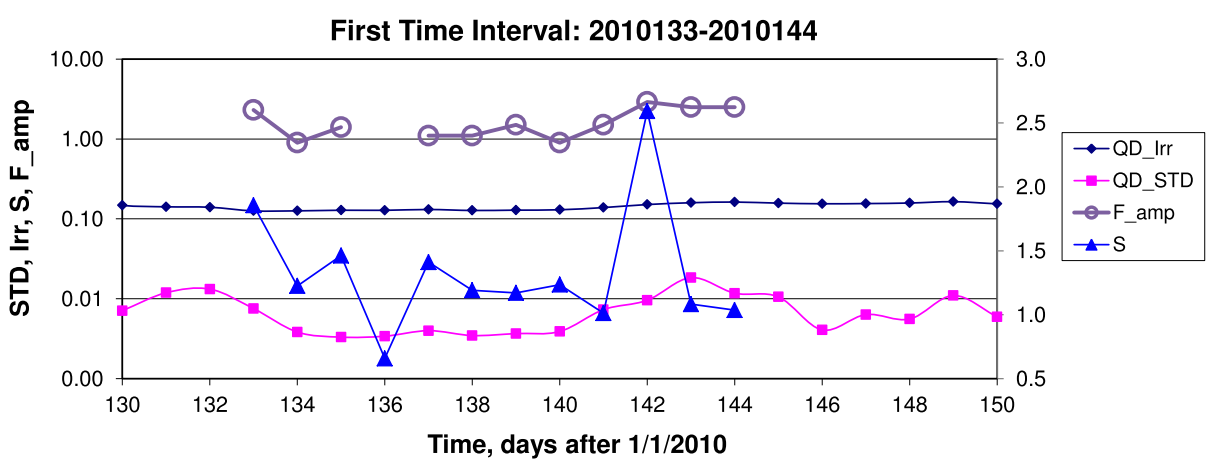

Figure 4 Time series for the first time interval used to analyze the correlation between the five-minute oscillation ratio (triangles), the daily mean irradiance (diamonds), and the STD of the irradiance (squares). Some of the positive correlations, e.g. between days 133-134 and 141-142, are changing to anti-correlations, for instance 140-141 and 142-143. The filter I2 (Equation (2)) maximum amplitudes (open circles) within the five-minute range are shown for the increases of the ratio $S>1.0$ (right scale). The gap in the maximum amplitude of filter $I 2$ (open circles) for day 136 in Figure 4 and other gaps in Figures 5 and 6 are related to the ratios with $S<1.0$ (Table 2, $S$-ratio) for which there is no power increase. These spectra show the noise in the five-minute region.

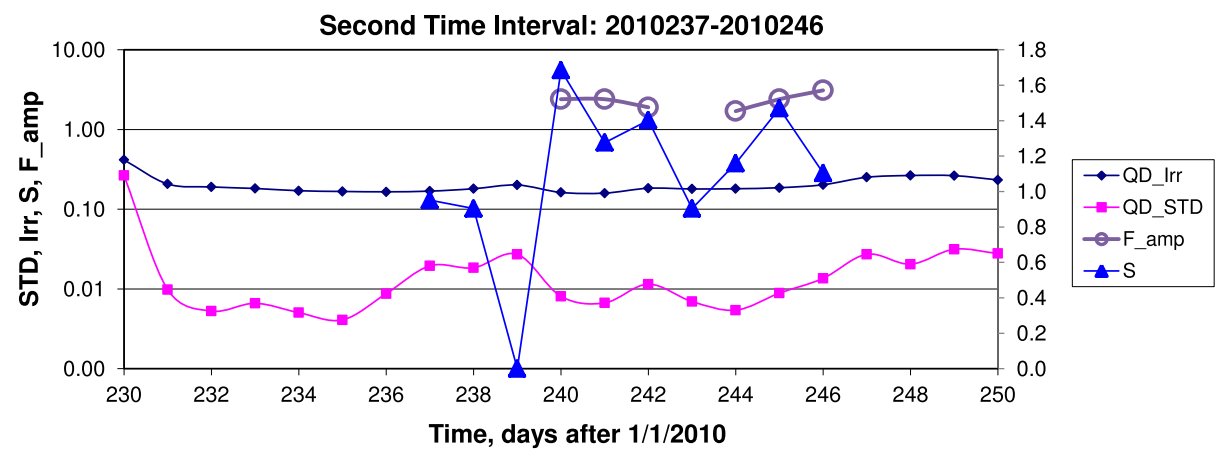

Figure 5 Time series for the second ten-day time interval (2010 DOY 237-246) used to analyze the correlation between the five-minute oscillation ratio (triangles), the daily mean irradiance (diamonds), and the STD of the irradiance (squares). Some positive correlations, e.g. between days 237-238, 240-241, and $242-243$, are changing to anti-correlations, for instance $238-239,243-244$, and 245-246. The filter I2 (Equation (2)) maximum amplitudes (open circles) within the five-minute range are shown for the increases of the ratio $S>1.0$ (right scale). The gaps in the maximum amplitude of filter $I 2$ (open circles) for days $237-239$, and 243 in Figure 5 are related to the ratios with $S<1.0$ (Table 2, $S$-ratio), for which there are no power increases. These spectra show the noise in the five-minute region.

Table 3 summarizes data from Table 2 for a more detailed comparison of the observing conditions.

The technique used for this analysis is based on a comparison of spectral amplitudes in the five-minute range of the power spectra. This technique is very sensitive to the contamination of the spectra by flare-related increases of the irradiance. These increases affect both the daily mean solar irradiance and the standard deviation [STD] of this irradiance. If the local five-minute increase in the power spectrum is a result of this contamination (flare-related solar noise), one should expect a positive correlation between the $S$-ratio (or maximum amplitude of filter I2) and the flare-related increases of the irradiance and its STD. Thus, the 


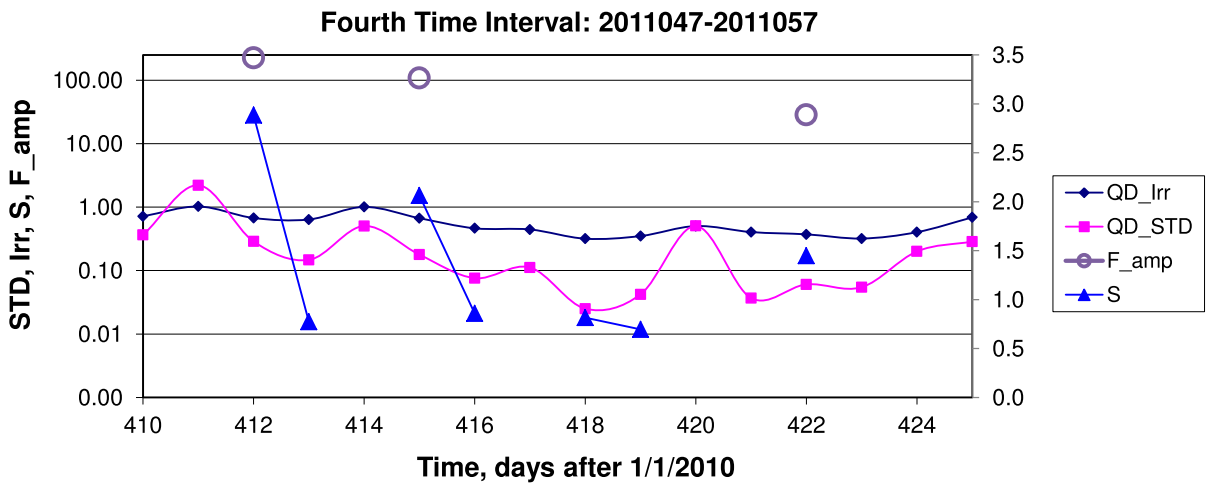

Figure 6 Time series for the fourth 11-day time interval (2011 DOY 47-57) shown as days $412-422$ used to analyze the correlation between the five-minute oscillation ratio (triangles), the daily mean irradiance (diamonds), and the STD of the irradiance (squares). Some positive correlations, e.g. between days $412-413$ and $415-416$, are changing to anti-correlations, for instance 418-419. The filter $I 2$ (Equation (2)) maximum amplitudes (open circles) within the five-minute range are shown for the increases of the ratio $S>1.0$ (right scale). The gaps in the maximum amplitude of filter $I 2$ (open circles) for days 413-414 and 416-421 in Figure 6 are related to the ratios with $S<1.0$ (Table 2, $S$-ratio) for which there is no power increase. These spectra show noise in the five-minute region.

Table 3 A comparison of the $S$-ratios in the five-minute band and their variations with the mean irradiance for each of the five time intervals.

\begin{tabular}{llll}
\hline $\begin{array}{l}\text { Days } \\
{[Y Y Y Y \text { DOY }]}\end{array}$ & $\begin{array}{l}\text { Mean irradiance } \\
10^{-1}\left[\mathrm{~mW} \mathrm{~m}^{-2}\right]\end{array}$ & $\begin{array}{l}\text { Mean STD } \\
10^{-3}\left[\mathrm{~mW} \mathrm{~m}^{-2}\right]\end{array}$ & $\begin{array}{l}\text { Mean } \\
\text { S-ratio }\end{array}$ \\
\hline $2010133-144$ & $1.37 \pm 1.35$ & $6.68 \pm 4.69$ & $1.09 \pm 0.12$ \\
$2010237-246$ & $1.81 \pm 1.45$ & $12.6 \pm 7.07$ & $1.03 \pm 0.05$ \\
$2010353-360$ & $1.60 \pm 0.07$ & $4.86 \pm 0.97$ & $0.92 \pm 0.07$ \\
$2011047-057$ & $5.31 \pm 2.02$ & $180 \pm 178$ & $1.04 \pm 0.12$ \\
$2011139-146$ & $2.76 \pm 0.15$ & $16.5 \pm 10.1$ & $0.99 \pm 0.05$
\end{tabular}

technical goal of this analysis was to extract and compare this information from the daily spectra for different levels of solar activity.

\subsubsection{Maximum Amplitude of Filter I2}

Figures 4-6 show the maximum amplitude of filter $I 2$ as open circles (see also last column in Table 2). For solar activity minimum conditions, the oscillation peaks in the power spectra are not masked by noise, and we assume that the maxima in the amplitude of filter $I 2$ for the first and second time intervals show the amplitudes of the five-minute oscillations in the corona related to the photospheric $p$-modes. For these conditions the mean amplitude is similar for the first and second time intervals: 1.69 and $2.16 \times 10^{-4}$ counts s$^{-1}$. The fourth time interval shows significantly higher amplitudes (Figure 6 and Table 2) that indicate the contamination of the spectra by much higher solar-flare activity. For low solar-activity periods, the maximum amplitude of filter $I 2$ is just another representation of the $S$-ratio, see Figure 4.

\subsubsection{Daily Mean Irradiance}

Table 2 and Figures 4-6 show that the daily mean soft X-ray irradiance is a significant source of the $S$-ratio change. Table 3 shows that the highest mean $S$-ratio of the oscillations 
in the five-minute band was detected for the first time interval with the lowest daily mean irradiance. The cross-correlation between the changes of the irradiance and the $S$-ratios for the first time interval is low at 0.1 . For the second time interval it becomes negative, -0.27 , which indicates that an increase of irradiance (1.81 compared to 1.37) leads to a decrease of $S$-ratios for the observed five-minute oscillations in the corona. For the fourth time interval, the correlation is positive and high at 0.64 . We interpret this as a contamination of the power spectra by solar-flare events. The amplitudes of the filter-curve maxima in Figures 4-6 (open circles) and the last column in Table 2 demonstrate such a flare-related increase. Because the spectral contamination is the result of the solar-flare low-frequency power transfer to the other frequency regions of the spectrum, including the five-minute region, we assume that it leads to the strong positive correlation detected for the fourth time interval.

\subsubsection{Standard Deviation}

The soft X-ray signal that ESP detects in the zeroth-order channel is a very sensitive probe of solar variability. Assuming that the observed five-minute oscillations in the corona represent a response of the corona to the photospheric acoustic modes and that the "transmission" of the solar atmosphere is a function of various disturbances and inhomogeneities between the photosphere and the corona, we can treat the standard deviation [STD] as an indicator of this solar "noise" in the five-minute oscillation signal. However, our results indicate that STD is not a unique parameter for estimating this transmission. For example, the cross-correlation between the $S$-ratio and STD for the first and second time intervals, 0.1 and -0.44 , is either low or negative. For the third time interval for which STD was the lowest (Table 3), the $S$ ratios were all $<1.0$ (Table 2). This may indicate that as suggested by several authors, e.g. Judge, Tarbell, and Wilhelm (2001), O'Shea, Muglach, and Fleck (2002), McIntosh, Fleck, and Judge (2003), Muglach (2003), and Vecchio et al. (2007), the connectivity between the photosphere and the corona depends on the configuration of the magnetic fields, which may also be a function of the STD. If the lowest STD during the third time interval is related to the decreased magnetic-field strength and to an ineffective configuration of the network, this may explain the absence of the power increases in the five-minute range of oscillations. The relatively high negative correlation $(-0.44)$ for the second time interval with an STD about two times higher compared to the first time interval allows us to consider that $S$ ratios for the second time interval as well as for the first time interval were not the result of the spectral contamination in the power spectra. This evidence is consistent with another independent confirmation of the response of the corona to photospheric $p$-modes, e.g. based on the spectral analysis of the two six-day time series (Didkovsky et al., 2011).

\subsubsection{A Shift of the Maximum Frequency as a Function of Activity}

We analyzed the oscillation spectra with the most significant power increases $(S \geq 1.1$, see Table $2, S$ ) in the five-minute range to investigate the correlation between the frequency of the maximum of this increase and solar activity (see Table 2, standard deviation). In addition to this correlation, the ranges of the increases were also analyzed. Table 4 and Figure 5 show the results of this analysis.

Figure 7 shows a positive correlation between the increases of the STD and the shift of the maximum frequency: $R_{1}=0.62$. The same correlation $\left(R_{2}=0.66\right)$ is found between the changes of STD and the mean frequency of the increase. Certainly, the significance of these correlations is low. Using the $t$-distribution, we find that $t=1.4$, which is lower 
Table 4 A comparison of the STD, the frequency maximum, and the ranges of power increases for parameter $S \geq 1.1$.

\begin{tabular}{lllll}
\hline $\begin{array}{l}\text { Days } \\
\text { [YYYY DOY] } \\
{\left[10^{-6} \mathrm{~W} \mathrm{~m}^{-2}\right]}\end{array}$ & $\begin{array}{l}\text { STD of the } \\
\text { irradiance } \\
{[\mathrm{mHz}]}\end{array}$ & $\begin{array}{l}\text { Max } \\
\text { frequency }\end{array}$ & $\begin{array}{l}\text { Range of } \\
\text { frequency } \\
{[\mathrm{mHz}]}\end{array}$ & $\begin{array}{l}\text { Mean } \\
\text { frequency } \\
{[\mathrm{mHz}]}\end{array}$ \\
\hline 2010133 & 7.54 & 3.0 & $2.0-4.1$ & 3.05 \\
2010135 & 3.31 & 2.4 & $2.1-4.1$ & 3.1 \\
2010137 & 3.99 & 3.0 & $2.6-3.5$ & 3.05 \\
2010142 & 9.58 & 3.5 & $2.5-5.3$ & 3.9 \\
2010240 & 8.09 & 2.7 & $2.1-4.4$ & 3.25 \\
2011047 & 288 & 3.6 & $2.4-5.4$ & 3.9 \\
\hline
\end{tabular}

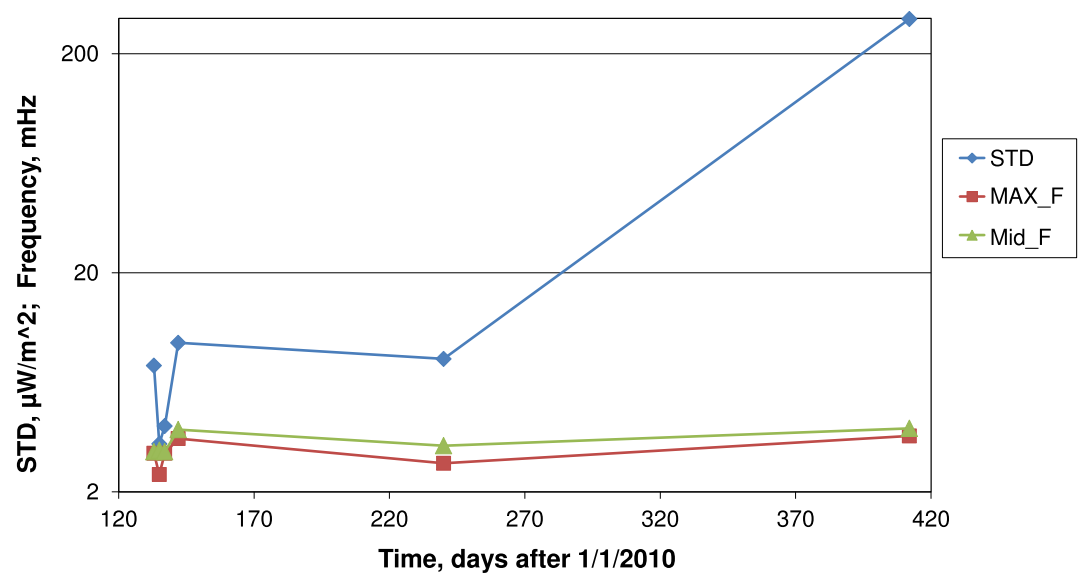

Figure 7 Comparison of the solar activity changes [STD: diamonds] with the changes of the frequency maximum increase [squares], and the mean frequency of the increase range [triangles]. Data for the power spectra are shown with increases of $S \geq 1.1$, see Table $2, S$-ratio.

than the $t$-distribution number of 1.638 for three degrees of freedom; the significance is 0.1 (one tail). Two days with the highest STD show a significant shift of the right edge of the frequency range toward the cut-off frequency, see 2010 DOY $142(5.3 \mathrm{mHz})$ and 2011 DOY $47(5.3 \mathrm{mHz})$ in Table 4. This shift is consistent with the model proposed by Bryson, Kosovichev, and Levy (2005).

\section{Concluding Remarks}

Global solar oscillations in the five-minute range were initially detected in the corona using soft X-ray irradiance measurements from SDO/EVE/ESP (Didkovsky et al., 2012). The variability of the five-minute $S$-ratios (Equation (3)) in the power spectra that characterize the strength of oscillations relative to noise was analyzed for five time intervals with lowand intermediate solar activity during a 379-day period (2010 DOY 133-2011 DOY 146) for 49 days.

The results of this analysis show that the best conditions for observing five-minute solar oscillations in the corona are at low solar activity. In the first time interval, 11 of the 12 days show power increases with an estimated $S$-ratio $\geq 1.0$. 
Our analysis shows that power increases in the spectra of coronal oscillations, interpreted as the response of the corona to photospheric $p$-modes due to their channeling (Vecchio et al., 2007) or leakage (Erdelyi et al., 2007; Malins and Erdelyi, 2007), are not related to an increased daily mean solar irradiance. This is clear from the $S$-ratio comparison between the first time interval and the other four time intervals, see Table 3 . The higher daily mean irradiances observed for the second through fifth time intervals did not lead to a higher $S$-ratio.

The power increases in the oscillation spectra are not caused by the increases in the mean STD (third column in Table 3) either. This conclusion is based on the analysis of cross-correlations between the $S$-ratio and the mean STD. The correlation is low for all time intervals except the fourth. This result confirms that detected increases of the $S$-ratios in the five-minute range are not created by the spectral contamination in the power spectra from solar flares and are not instrumental or data-reduction artifacts, but represent the leakage of photospheric $p$-modes to the corona.

We interpret the significant positive correlation for the fourth time interval as an artifact and a reflection of much higher solar activity with an STD about 27 times higher than for the first time interval. The amplitude maxima of filter $I 2$ for the fourth time interval are significantly larger than the amplitudes for the first and second time intervals and may be a demonstration of the spectral contamination in the five-minute region by the low-frequency flare signals. This is also clear from helioseismology results, which have shown shifts in the frequencies of the global modes related to the solar cycle, but not in the amplitudes.

Another conclusion based on these results is that large-scale solar oscillations detected in the corona are related to the leakage of the photospheric acoustic oscillations rather than to the excitation of these coronal oscillations by solar energetic events.

The high sensitivity of the five-minute oscillations in the corona to the changes of solar irradiance may be used as a diagnostic tool to characterize the connectivity in soft X-ray irradiance dynamics.

Acknowledgements This work was partially supported by the University of Colorado award 153-5979. Data courtesy of NASA/SDO and the EVE science team.

Open Access This article is distributed under the terms of the Creative Commons Attribution License which permits any use, distribution, and reproduction in any medium, provided the original author(s) and the source are credited.

\section{References}

Andersen, B.N.: 1991, Adv. Space Res. 11, 93.

Andreev, A.S., Kosovichev, A.G.: 1995, In: Hoeksema, J.T., Domingo, V., Fleck, B., Battrick, B. (eds.) $S O H O$ 4 - Helioseismology SP-376, ESA, 471.

Andreev, A.S., Kosovichev, A.G.: 1998, In: Wilson, A. (ed.) SOHO 6/GONG - Structure and Dynamics of the Interior of the Sun and Sun-like Stars SP-418, ESA, Noordwijk, 87.

Bryson, S., Kosovichev, A., Levy, D.: 2005, Physica D 201, 1.

Chaplin, W.J., Elsworth, Y., Isaak, G.R., Lines, R., McLeod, C.P., Miller, B.A., New, R.: 1998, Mon. Not. Roy. Astron. Soc. 300, 1077.

Claverie, A., Isaak, G.R., McLeod, C.P., Van Der Raay, H.B., Roca Cortes, T.: 1979, Nature 282, 591.

De Pontieu, B., Erdelyi, R., James, S.P.: 2004, Nature 430, 536.

Didkovsky, L.V., Judge, D.L., Wieman, S., Harmon, M., Woods, T., Jones, A., Chamberlin, P., Woodraska, D., Eparvier, F., McMullin, D., Furst, M., Vest, R.: 2007, In: Fineschi, S., Viereck, A.R. (eds.) Solar Physics and Space Weather Instrumentation II, SPIE 6689, 6689OP1.

Didkovsky, L., Judge, D., Kosovichev, A., Wieman, S., Woods, T.: 2011, Astrophys. J. Lett. 738 , L7.

Didkovsky, L., Judge, D., Wieman, S., Woods, T., Jones, A.: 2012, Solar Phys. 275, 179. 
Erdelyi, R., Malins, C., Toth, G., De Pontieu, B.: 2007, Astron. Astrophys. 467, 1299.

Fröhlich, C., Andersen, B.N., Appourchaux, T., Berthomieu, G., Crommelynck, D.A., Domingo, V., Fichot, A., Finsterle, W., Gomez, M.F., Gough, D., Jemenez, A., Leifsen, T., Lombaerts, M., Pap, J.M., Provost, J., Cortes, T.R., Romero, J., Roth, H., Sekii, T., Telljohann, U., Toutain, T., Wehrli, C.: 1997, Solar Phys. 170, 1. ADS:1997SoPh..170....1F, doi:10.1023/A:1004969622753.

Goode, P.R., Gough, D., Kosovichev, A.G.: 1992, Astrophys. J. 387, 707.

Gough, D.O.: 1993, In: Zahn, J.-P., Zinn-Justin, J. (eds.) Astrophysical Fluid Dynamics - Les Houches Session XLVII, 1987, Elsevier, Amsterdam, 399.

Gurman, J.B., Leibacher, J.W., Shine, R.A., Woodgate, B.E., Henze, W.: 1982, Astrophys. J. $253,939$.

Hovestadt, D., Hilchenbach, M., Burgi, A., Klecker, B., Laeverenz, P., Scholer, M., Grunwaldt, H., Axford, W.I., Livi, S., Marsch, E., Wilken, B., Winterhoff, H.P., Ipavich, F.M., Bedini, P., Coplan, M.A., Galvin, A.B., Gloeckler, G., Bochsler, P., Balsiger, H., Fisher, J., Geiss, J., Kallenbach, R., Wurz, P., Reiche, K.-U., Gliem, F., Judge, D.L., Ogawa, H.S., Hsieh, K.C., Mobius, E., Lee, M.A., Managadze, G.G., Verigin, M.I., Neugebauer, M.: 1995, Solar Phys. 162, 441. ADS:1995SoPh..162..441H, doi:10.1007/BF00733436.

Judge, D.L., McMullin D, R., Ogawa, H.S., Hovestadt, D., Klecker, B., Hilchenbach, M., Mobius, E., Canfield, L.R., Vest, R.E., Watts, R., Tarrio, C., Kuhne, M., Wurz, P.: 1998, Solar Phys. 177, 161. ADS:1998SoPh..177..161J, doi:10.1023/A:1004929011427.

Judge, P.G., Tarbell, T.D., Wilhelm, K.: 2001, Astrophys. J. 554, 424.

Malins, C., Erdelyi, R.: 2007, Solar Phys. 246, 41. ADS:2007SoPh..246...41M, doi:10.1007/s11207-0079073-8.

McIntosh, S.W., Fleck, B., Judge, P.G.: 2003, Astron. Astrophys. 405, 769.

Muglach, K.: 2003, Astron. Astrophys. 401, 685.

O’Shea, E., Muglach, K., Fleck, B.: 2002, Astron. Astrophys. 387, 642.

Vecchio, A., Gauzzi, G., Reardon, K.P., Janssen, K., Rimmele, T.: 2007, Astron. Astrophys. 461, L1.

Woods, T.N., Eparvier, F.G., Hock, R., Jones, A., Woodraska, D., Judge, D., Didkosky, L., Lean, J., Mariska, J., Warren, H., McMullin, D., Chamberlin, P., Berthiaume, G., Bailey, S., Fuller-Rowell, T., Soika, J., Tobiska, W.K., Viereck, R.: 2012, Solar Phys. 275, 115. ADS:2012SoPh..275..115W, doi:10.1007/s11207-009-9487-6. 